\title{
Analysis of Factors Affecting Functional Outcomes in Robotic-assisted Laparoscopic Radical Prostatectomy
}

\author{
(1) Fuat Kızılay MD, (1) Fuad İsmaylov MD, (1) Adnan Şimşir MD, (1) Burak Turna MD, (1) Bülent Semerci MD, (1) Erdal Apaydın MD \\ Ege University Faculty of Medicine, Department of Urology, Izmir, Turkey
}

\begin{abstract}
Objective: In addition to ensuring cancer control, prevention of incontinence and erectile dysfunction, which significantly impact patients' quality of life, is also an important issue in robot-assisted laparoscopic radical prostatectomy (RALRP) operations. In this study, we aimed to evaluate the factors affecting postoperative urinary continence and erectile function in patients who underwent RALRP due to localized prostate cancer in our clinic.

Materials and Methods: Our study included 439 patients who were diagnosed with stage 1 prostate cancer and underwent RALRP. Patients' age, preoperative prostate-specific antigen (PSA) value, prostate volume, radical prostatectomy material Gleason score, operative time, transperitoneal surgical approach (posterior or anterior), and surgical margin and extraprostatic extension statuses were recorded. Postoperative continence and erectile function status of the patients were questioned and recorded via telephone interviews and in outpatient clinic follow-up. Patients were divided into groups according to postoperative incontinence and erectile dysfunction status and the variables were compared between the groups.

Results: There was no statistically significant difference between the continent and incontinent groups in terms of age, preoperative PSA, prostate volume, operative time, postoperative Gleason score, surgical margin status, extraprostatic extension status, or anterior or posterior approach ( $p>0.05$ ). There was no statistically significant difference between the groups with and without erectile dysfunction in terms of prostate volume, operative time, postoperative Gleason score, surgical margin status, or extraprostatic extension status $(p>0.05)$, while there were statistically significant differences between the 2 groups in terms of age $(p<0.001)$, preoperative PSA value $(p=0.042)$, and surgical technique $(p<0.001)$.

Conclusion: We concluded that patient- and disease-related factors did not significantly affect postoperative urinary continence in patients undergoing RALRP due to prostate cancer, while patient age, preoperative PSA value, and operative technique had a significant effect on erectile function.
\end{abstract}

Keywords: Robot-assisted laparoscopic radical prostatectomy, prostate cancer, erectile dysfunction, incontinence, quality of life

\section{Introduction}

Globally, prostate cancer $(\mathrm{PCa})$ is the $4^{\text {th }}$ most common type of cancer among both genders and second most common among males (1). PCa is often diagnosed in young and healthy men, and in addition to providing long-term cancer control, preserving patients' quality of life is also an important goal. Radical prostatectomy (RP) is considered the gold standard for surgical treatment of localized PCa (2). RP outcomes are generally assessed in terms of urinary continence, potency, and cancer control, referred to as the "trifecta" (3). After Walsh and Donker (4) developed the anatomic nerve-sparing technique for retropubic RP (RRP), RRP became the gold standard, most widely used surgical method that provides excellent cancer control for clinically localized PCa (5).

A minimally invasive method for PCa treatment aiming to reduce the morbidity of RRP was first described in 1992 by Schuessler et al. (6). The authors concluded that laparoscopic RP (LRP) was 
a difficult technique with a long learning curve and offered no advantages over RRP. Larger series published later showed the technique to be feasible with similar outcomes to open surgery $(7,8)$. However, its technical difficulty and long learning curve prevented it from being widely accepted among surgeons.

Bringing technological advances such as 3-dimensional (3D) imaging, 7 degrees of freedom, surgical comfort, and extension of surgical field, the introduction of the Da Vinci Robotic Surgical System (Intuitive Surgical, Inc., Sunnyvale, CA) offered a groundbreaking minimally invasive method for RP. About a decade after robot-assisted LRP (RALRP) was introduced, many modifications were made and the technique was standardized $(8,9)$. Data from large series indicate that RALRP yields similar oncological results as other large RRP and LRP series. The introduction of this technique resulted in greater patient expectations, and complications and surgical margin status were added to the trifecta to create a pentafecta.

The aim of the present study was to analyze parameters that affect urinary continence and erectile function, the main factors determining postoperative quality of life, in patients who underwent RALRP in our clinic for localized PCa.

\section{Materials and Methods}

\section{Patient Selection and Data Collection}

Data from 439 patients who were diagnosed with stage 1 PCa and treated with RALRP in our clinic between March 2012 and January 2017 were retrospectively analyzed. The patients' age, preoperative prostate-specific antigen (PSA) level, prostate volume, Gleason score of RP material, surgery duration, transperitoneal RALRP technique (posterior or anterior approach), surgical margin positivity, and extraprostatic spread were noted. Written consent was obtained from the patients prior to the operation. The patients were invited for followup visits by telephone and were assessed for postoperative continence and erectile function. Those who did not use pads or those who used a single pad for protection per 24 hours were considered continent. Erectile function was evaluated using the International Index of Erectile Function (IIEF) and patients with an IEFF scores below 10 were accepted as having erectile dysfunction. Patients with at least 12 months of postoperative follow-up were included in the study.

Inclusion criteria for the study were being diagnosed with PCa by transrectal ultrasound-guided biopsy or transurethral prostate resection, and having prostate-limited disease (stage 1). Exclusion criteria were presence of preoperative erectile dysfunction, preoperative urinary incontinence, or locally advanced or advanced PCa.

\section{Statistical Analysis}

Statistical analysis of the data were done using SPSS 17.0 software package. Study data were expressed as mean \pm standard deviation or number (percent). Student's t-test and chi-square tests were used for statistical analyses. $P$ value $<0.05$ was considered statistically significant.

\section{Robot-Assisted Laparoscopic Radical Prostatectomy Procedure}

A $12 \mathrm{~mm}$ trocar was placed superior to the umbilicus. A point approximately $15 \mathrm{~cm}$ superior to the symphysis pubis and about 7-8 $\mathrm{cm}$ left lateral was marked and a second $8 \mathrm{~mm}$ trocar was placed 7-8 $\mathrm{mm}$ lateral to the first one. These trocars were used for robotic arms 2 and 3, respectively. An $8 \mathrm{~mm}$ trocar was then inserted $7-8 \mathrm{~cm}$ lateral to the reference mark. This included robotic arm 1. A $12 \mathrm{~mm}$ trocar was inserted $3-4 \mathrm{~cm}$ superomedial to the iliac crest on the axis directly connecting the iliac crest and the camera port. Finally, the $5 \mathrm{~mm}$ assistant port was inserted between the 2 previously placed right ports, about $3 \mathrm{~cm}$ superior to the line connecting the 2 trocars. An incision was made in the peritoneum above the symphysis pubis level. The median umbilical ligaments and urachus were cut. After cutting the endopelvic facia, sutures were made around the dorsal vein complex with 0-PDS or Vicryl on a CT-1 needle. The Foley catheter was deflated and withdrawn to the urethra to visualize the vesical trigone. When the posterior bladder and the trigone were clearly visible, the incision was continued along the length of the bladder neck.

Two approaches have been described for the dissection of seminal vesicles in transperitoneal RALRP: posterior (Montsouris technique) and anterior (Menon technique). In the anterior approach (Menon technique), seminal vesicles are located and dissected after the prostate and the posterior bladder neck incision. The posterior approach is essentially the dissection of the vas deferens and seminal vesicles before developing the Retzius space. In this approach, retrovesical antegrade dissection of the vas deferens and seminal vesicles was done first. A U-shaped incision was made in the peritoneum 1-1.5 $\mathrm{cm}$ above the rectum over the vasa deferentia. The areolar tissue in the region was dissected in order to locate and dissect the vasa deferentia. The seminal vesicles posterior to the vasa deferentia were also located and separated from the surrounding tissues by blunt and sharp dissection. The fascial sheath around the prostate was dissected. The lateral pelvic fascia was sharply incised along the anterolateral prostate. It was temporarily occluded using Weck clips and sutured after removal of the prostate. The ipsilateral seminal vesicle was grasped with arm 4 and suspended to clearly expose the pedicle. After cutting the pedicle, the posterolateral connections between the neurovascular bundle and the prostate were sharply incised with scissors. The catheter was withdrawn and the posterior urethra was cut. The surgical specimen was then removed and placed into a laparoscopy bag or left in the pelvis. A secure, mucosa-to-mucosa, vesicorurethral anastomosis was formed using continuous suture. After the anastomosis was created, a Foley catheter was inserted and the bladder was filled to check for anastomotic leakage. The stages of RALRP are summarized in Figure 1.

The patients were discharged on postoperative day 3 or 4 . Foley catheters were removed on postoperative day 7 .

\section{Results}

The patients' mean age was $64.29 \pm 6.69$ years, mean PSA value was $9.52 \pm 10.25 \mathrm{ng} / \mathrm{mL}$, mean prostate volume was $48.28 \pm 19.86 \mathrm{~mL}$, and mean surgery duration was $146.18 \pm 25.90$ 
min. Postoperatively, 273 (62.2\%) of the 439 patients did not experience incontinence while 166 (37.8\%) patients did. Sixty-seven patients with preoperative erectile dysfunction were excluded from the study, leaving 372 patients included in the study for analysis of postoperative erectile dysfunction. Postoperatively, $173(46.5 \%)$ of the 372 patients had no erectile dysfunction while 199 (53.5\%) patients experienced erectile dysfunction.

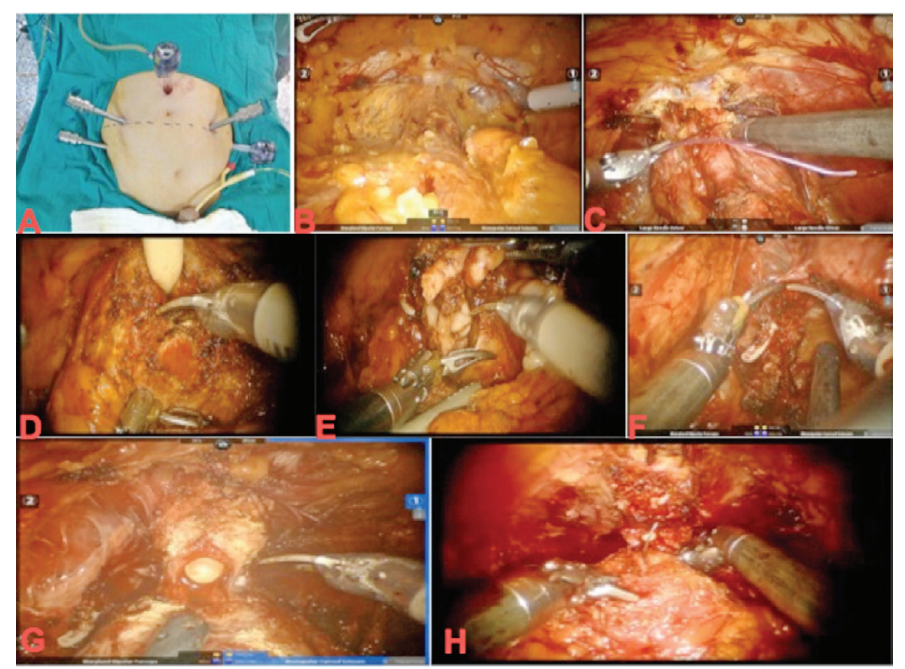

Figure 1. Stages of robot-assisted laparoscopic radical prostatectomy: A) placement of ports for robot-assisted radical prostatectomy, B) development of the Retzius space to expose the prostate, $C$ ) tying the deep dorsal vein plexus, D) dissection of the bladder neck, E) dissection of the vasa deferentia and seminal vesicles, F) dissection of the neurovascular bundle, $\mathrm{G}$ ) dissection of the urethra, $\mathrm{H}$ ) creation of vesicourethral anastomosis

Table 1. Comparison of age, prostate-specific antigen, prostate volume, surgery duration, surgical technique, surgical margin, extraprostatic extension, and Gleason score of prostatectomy material in patients with and without postoperative incontinence

\begin{tabular}{|c|c|c|c|}
\hline & \multicolumn{2}{|l|}{ Incontinence } & \multirow{2}{*}{$p$} \\
\hline & No & Yes & \\
\hline Age (years) & $63.84 \pm 6.51$ & $65.05 \pm 6.93$ & $0.067^{1}$ \\
\hline PSA (ng/mL) & $8.97 \pm 9.10$ & $10.42 \pm 11.8$ & $0.152^{1}$ \\
\hline Prostate weight (g) & $47.60 \pm 18.7$ & $49.43 \pm 21.3$ & $0.347^{1}$ \\
\hline Operative time (minutes) & $148.22 \pm 24.9$ & $142.83 \pm 24.8$ & $0.028^{1}$ \\
\hline Postoperative Gleason score & $7.03 \pm 0.60$ & $6.98 \pm 0.68$ & $0.413^{1}$ \\
\hline Anterior approach & $173(64.3 \%)$ & $96(35.7 \%)$ & \multirow{2}{*}{$0.248^{2}$} \\
\hline Posterior approach & $100(58.8 \%)$ & $70(41.2 \%)$ & \\
\hline Negative surgical margin & $179(63.3 \%)$ & $104(36.7 \%)$ & \multirow[t]{2}{*}{$0.539^{2}$} \\
\hline Positive surgical margin & $94(60.3 \%)$ & $62(39.7 \%)$ & \\
\hline Extraprostatic spread (-) & $175(65.8 \%)$ & $91(34.2 \%)$ & \multirow[t]{2}{*}{$0.056^{2}$} \\
\hline Extraprostatic spread (+) & $98(56.6 \%)$ & $75(43.4 \%)$ & \\
\hline \multicolumn{4}{|c|}{$\begin{array}{l}\text { PSA: Prostate-specific antigen } \\
\text { 'Student's t-test } \\
{ }^{2} \text { Chi-square test } \\
\text { Values are given as mean } \pm \text { standard deviation or number (percent) }\end{array}$} \\
\hline
\end{tabular}

There were no statistical differences between patients with and without postoperative incontinence in terms of demographical data, surgery duration, perioperative data, or postoperative histopathological data. The findings are summarized in Table 1. Age $(p<0.001)$, preoperative PSA value $(p=0.042)$, and anterior or posterior approach in RALRP operation $(p<0.001)$ were significant factors influencing the incidence of postoperative erectile dysfunction. However, the other demographic data, prostate characteristics, and histopathological parameters did not have a significant impact on erectile function. The correlations between the variables and postoperative erectile function are shown in Table 2.

\section{Discussion}

RALRP is one of the surgical techniques that developed rapidly and became widespread in the field of urology following the worldwide introduction of robotic systems. Various factors have made robotic methods feasible and appealing for RP, including the ability to have a closer, 3D view of the surgical site, make a sharper apical dissection, have a greater urethral length and more effective nerve sparing, reduced incisional morbidity, reduced need for blood transfusion and analgesic, and shorter hospital stay and recovery time.

While surgical treatment of PCa primarily targets oncological control, ensuring continued continence and erectile function is also important for the patient's quality of life.

Urinary incontinence is certainly one of the most important post-RALRP complications that impacts patients' daily lives. Full continence is defined in the literature as patients not using incontinence pads at all or only using a pad for security.

Many studies have investigated the effect of prostate volume on post-RP continence. Choo et al. (10) found that the likelihood of regaining continence in patients with a prostate larger than $40 \mathrm{~g}$ was lower in the RALRP group than in the group that had

Table 2. Relationship between demographic, surgical, and histopathological data and postoperative erectile dysfunction

\begin{tabular}{|c|c|c|c|}
\hline & \multicolumn{2}{|c|}{ Erectile dysfunction } & \multirow{2}{*}{ p } \\
\hline & No & Yes & \\
\hline Age (years) & $61.44 \pm 6.00$ & $64.59 \pm 6.05$ & $<0.001^{1}$ \\
\hline PSA (ng/mL) & $8.28 \pm 7.44$ & $10.18 \pm 10.5$ & $0.042^{1}$ \\
\hline Prostate weight (g) & $47 \pm 18.8$ & $49 \pm 20.3$ & $0.328^{1}$ \\
\hline Surgery duration (minutes) & $143 \pm 24.9$ & $147 \pm 24.2$ & $0.061^{1}$ \\
\hline Postoperative Gleason score & 7.05 & 6.98 & $0.330^{1}$ \\
\hline Anterior approach & $73(34.4 \%)$ & $139(65.6 \%)$ & \multirow[t]{2}{*}{$<0.001^{2}$} \\
\hline Posterior approach & $100(62.5 \%)$ & $60(37.5 \%)$ & \\
\hline Negative surgical margin & $110(44 \%)$ & $140(56 \%)$ & \multirow[t]{2}{*}{$0.248^{2}$} \\
\hline Positive surgical margin & $63(51.6 \%)$ & $59(48.4 \%)$ & \\
\hline Extraprostatic spread (-) & $111(48.1 \%)$ & $120(51.9 \%)$ & \multirow[t]{2}{*}{$0.455^{2}$} \\
\hline Extraprostatic spread (+) & $62(44 \%)$ & $79(56 \%)$ & \\
\hline \multicolumn{4}{|c|}{$\begin{array}{l}\text { PSA: Prostate-specific antigen } \\
\text { 'Student's t-test } \\
{ }^{2} \text { Chi-square test } \\
\text { Values are given as mean } \pm \text { standard deviation or number (percent) }\end{array}$} \\
\hline
\end{tabular}


open RP ( $97 \%$ vs $88 \%, p=0.025)$. The group with small prostate volume also had better outcomes with regard to potency (56\% vs $50 \%, p=0.614)(10)$. Similarly, in a study evaluating the effects of prostate volume on functional outcomes, Boczko et al. (11) separated 355 patients who underwent RALRP into 2 groups, those with prostate volume greater than $75 \mathrm{~g}(\mathrm{n}=36)$ and less than $75 \mathrm{~g}(\mathrm{n}=319)$, for comparison. At 6 months, the continence rate was $97 \%$ in the group with lower prostate volume and $84 \%$ in the group with higher volume $(p<0.05)$ (11). In addition to studies showing that smaller prostate volume has a favorable impact on post-RALRP urinary continence, the literature also includes studies in which prostate volume was not a significant factor in continence $(12,13,14)$. Similar to the work of Labanaris et al. (13) and Yasui et al. (14) the results of our study suggest that prostate volume is not a determining factor in postoperative continence. Prostate volume was not a determinant of potency either.

Another factor investigated for its effect on post-RALRP continence is patient age. Kumar et al. (15) followed patients below and above the age of 70 with similar clinicopathologic characteristics (400 in each group) for 2 years and found similar continence rates $(p=0.06)$ and time to regain continence. As for potency, both postoperative potency rates and time to regain potency were better in the younger group (15). Greco et al. (16) also divided patients into those younger and older than 70 and found that older males had significantly lower continence rates at postoperative 6 months but improved to a level comparable to that of the younger males by postoperative 12 months. However, it should be noted that the study included a significantly smaller number of older males compared to younger ones (23 vs 180). Similarly, Novara et al. (17) and Kim et al. (18) found that patient age was an independent prognostic factor for postoperative recovery of continence. Zorn et al. (19) divided patients into those older and younger than 60 and reported that continence rates were not different at 1-year follow-up after RALRP, but the younger patients had better results in terms of potency. Similar to their study, we also observed no significant association between mean age and continence in the present study, whereas the group exhibiting postoperative potency was younger. Advanced age was determined to be an important parameter in the development of postoperative erectile dysfunction. Another study investigated the effects of prostate volume and age on early post-RALRP recovery of erectile function. Of the 139 patients in the study, 53 showed subjective potency recovery at 3 months. In univariate analysis, prostate weight (43.3 vs 51.4 $g, p=0.038$ ) and age (55 vs 57 years, $p=0.03$ ) were significant. In multivariate analysis, only prostate weight was significantly correlated with potency $(p=0.03)(20)$. Mendiola et al. (21) reported that younger males were more likely to regain potency in the early period than older males. In their study, patients were divided into 3 age groups: $<50$ years, $50-59$ years, and $\geq 60$ years. Young males (below 50) regained subjective potency earlier than the older patients $(p=0.01)$. Potency rates were significantly higher among the young males at 3 and 6 months $(p=0.04$ for both) and this trend continued until 12 months (21).

There are various studies in the literature concerning the effects of PSA level on post-RALRP functional outcomes. Xylinas et al.
(22) examined postoperative continence and erectile function in 500 patients who underwent RALRP. The patients' median PSA level was $9.7 \mathrm{ng} / \mathrm{mL}$ and median age was 62.2 , and rates of continence and potency at 1 and 2 years were $44 \%$ and $53 \%$, respectively. The authors reported that PSA below $10 \mathrm{ng} /$ $\mathrm{mL}$ and age younger than 60 were correlated with favorable early functional outcomes (22). On the other hand, Torer et al. (23) found that preoperative serum PSA and Gleason score did not affect continence in 385 patients operated for RALRP. In our study, we found no significant difference in PSA level between patients with and without postoperative urinary incontinence. Our patients exhibited a higher rate of erectile dysfunction with higher PSA values $(p=0.042)$. These data are consistent with the literature.

Another subject of analysis is the effect of the RALRP technique on functional results. In a study by Ko et al. (24), patients who underwent anterior $(n=172)$ and posterior $(n=172)$ RALRP were analyzed in separate groups. The potency ratios at 3, 6, and 9 months were $80.8 \%, 90.1 \%$, and $92.9 \%$ respectively in the posterior group and $65 \%, 72.1 \%$, and $85.3 \%$ respectively in the anterior group. At 12 months, there was no difference in potency rates between the groups, though it was noted that the posterior method resulted in significantly higher rates of early potency recovery. No significant difference was found between the methods with regard to continence rates or time to continence recovery (24). We also observed no significant difference between patients operated via the anterior and posterior approach with regard to incontinence. However, the posterior group exhibited less erectile dysfunction. Based on our findings, the effect of surgical technique on RALRP functional outcomes were similar to that reported by Ko et al. (24). On the other hand, Maddox et al. (25) had different results due to the shorter follow-up period.

\section{Study Limitations}

Limitations of this study include that it is retrospective, the RALRP procedures were performed by different surgeons, and there was no control group.

\section{Conclusion}

Technological advances and innovations in RALRP surgery have occurred in parallel with the ongoing development of robotic systems from their first incarnations, accumulation of knowledge regarding the anatomic and pathological characteristics of $\mathrm{PCa}$, and experience gained through the widespread use of the technique. Our findings in this study indicate that patientand disease-related factors are not associated with post-RALRP urinary continence, while erectile function is mainly influenced by the patient's age, preoperative PSA value, and the surgical technique. Conflicting functional outcomes reported after RALRP may be attributable to differences in patient populations, the questionnaire forms used, and the lack of standardization of the surgical techniques.

\section{Ethics}

Ethics Committee Approval: Retrospective study. 
Informed Consent: Written consent was obtained from the patients prior to the operation.

Peer-review: Externally peer-reviewed.

\section{Authorship Contributions}

Surgical and Medical Practices: F.K., F.l., A.Ş., B.T., B.S., E.A., Concept: F.K., F.I., E.A., Design: F.K., F.I., E.A., Data Collection or Processing: F.K., F.I., E.A., Analysis or Interpretation: F.K., F.I., A.Ş., B.T., B.S., E.A., Literature Search: F.K., F.l., Writing: F.K., F.l.

Conflict of Interest: No conflict of interest was declared by the authors.

Financial Disclosure: The authors declared that this study received no financial support.

\section{References}

1. Ferlay J, Shin HR, Bray F, et al. Estimates of worldwide burden of cancer in 2008: GLOBOCAN 2008. Int J Cancer 2010;127:2893-2917.

2. Bill-Axelson $A$, Holmberg L, Filén F, et al. Radical prostatectomy versus watchful waiting in localized prostate cancer: the Scandinavian prostate cancer group-4 randomized trial. J Natl Cancer Inst 2008; 100:1144-1154.

3. Eastham JA, Scardino PT, Kattan MW. Predicting an optimal outcome after radical prostatectomy: the trifecta nomogram. J Urol 2008; 179:2207-2210.

4. Walsh PC, Donker PJ. Impotence Following Radical Prostatectomy: Insight into Etiology and Prevention. J Urol 2017;197:165-170.

5. Cabo AV, Nguyen DP, Touijer K. Surgical Management of Localized and Locally Advanced Prostate Cancer. In: Merseburger AS, Burger M, eds. Urol Oncol. Cham: Springer International Publishing; 2017. P. 1-19.

6. Schuessler WW, Schulam PG, Clayman RV, Kavoussi LR. Laparoscopic radical prostatectomy: initial short-term experience. Urology 1997; 50:854-857.

7. Rassweiler J, Sentker L, Seemann O, et al. Laparoscopic radical prostatectomy with the Heilbronn technique: an analysis of the first 180 cases. J Urol 2001;166:2101-2108.

8. Eden CG, Cahill D, Vass JA, et al. Laparoscopic radical prostatectomy: the initial UK series. Bju Int 2002;90:876-882.

9. Pasticier G, Rietbergen JB, Guillonneau B, et al. Robotically assisted laparoscopic radical prostatectomy: feasibility study in men. Eur Urol 2001;40:70-74.

10. Choo MS, Choi WS, Cho SY, et al. Impact of prostate volume on oncological and functional outcomes after radical prostatectomy: robot-assisted laparoscopic versus open retropubic. Korean J Urol 2013;54:15-21.
11. Boczko J, Erturk E, Golijanin D, et al. Impact of prostate size in robotassisted radical prostatectomy. J Endourol 2007;21:184-188.

12. Boylu U, Turan T, Basatac C, et al. The effect of prostate weight on the outcomes of robot-assisted radical prostatectomy. Turk J Urol 2013;39:209-213.

13. Labanaris AP, Zugor V, Witt JH. Robot-assisted radical prostatectomy in patients with a pathologic prostate specimen weight $\geq 100$ grams versus $\leq 50$ grams: surgical, oncologic and short-term functional outcomes. Urol Int 2013;90:24-30.

14. Yasui T, Tozawa K, Kurokawa $S$, et al. Impact of prostate weight on perioperative outcomes of robot-assisted laparoscopic prostatectomy with a posterior approach to the seminal vesicle. BMC Urol 2014;14:6.

15. Kumar A, Samavedi S, Bates AS, et al. Age stratified comparative analysis of perioperative, functional and oncologic outcomes in patients after robot assisted radical prostatectomy--A propensity score matched study. Eur J Surg Oncol 2015;41:837-843.

16. Greco KA, Meeks IJ, Wu S, et al. Robot-assisted radical prostatectomy in men aged $>$ or $=70$ years. Bju Int 2009;104:1492-1495.

17. Novara G, Ficarra V, D’Elia C, et al. Evaluating urinary continence and preoperative predictors of urinary continence after robot assisted laparoscopic radical prostatectomy. J Urol 2010;184:1028-1033.

18. Kim SC, Song C, Kim W, et al. Factors determining functional outcomes after radical prostatectomy: robot-assisted versus retropubic. Eur Urol 2011;60:413-419.

19. Zorn KC, Mendiola FP, Rapp DE, et al. Age-stratified outcomes after robotic-assisted laparoscopic radical prostatectomy. J Robot Surg 2007; 1:125-132.

20. Ahlering TE, Kaplan AG, Yee DS, et al. Prostate weight and early potency in robot-assisted radical prostatectomy. Urology 2008;72:1263-1268.

21. Mendiola FP, Zorn KC, Mikhail AA, et al. Urinary and sexual function outcomes among different age groups after robot-assisted laparoscopic prostatectomy. J Endourol 2008;22:519-524.

22. Xylinas E, Durand X, Ploussard G, et al. Evaluation of combined oncologic and functional outcomes after robotic-assisted laparoscopic extraperitoneal radical prostatectomy: trifecta rate of achieving continence, potency and cancer control. Urol Oncol 2013;31:99-103.

23. Torer BD, Eksi M, Kargi T, et al. Retrospective Analysis of Factors Affecting Continence after Robotic Radical Prostatectomy. J Acad Res Med 2017;7:21-25.

24. Ko YH, Coelho RF, Sivaraman A, et al. Retrograde versus antegrade nerve sparing during robot-assisted radical prostatectomy: which is better for achieving early functional recovery? Eur Urol 2013;63:169-177.

25. Maddox M, Elsamra S, Kaplon D, et al. The posterior surgical approach to robot-assisted radical prostatectomy facilitates dissection of large glands. J Endourol 2013;27:740-742. 